\title{
Regulation of water resources systems using fuzzy logic: a case study of Amaravathi dam
}

\author{
P. V. P. Moorthi ${ }^{1} \cdot$ Ajit Pratap Singh $^{1} \mathbb{D} \cdot$ P. Agnivesh ${ }^{1}$
}

Received: 16 September 2016 / Accepted: 31 July 2018 / Published online: 6 August 2018

(c) The Author(s) 2018

\begin{abstract}
Regulation of a water resource system is one of the challenging tasks due to uncertainty involved in demand and supply. It may be due to changes in the climatic conditions, living standards of people, land-use patterns and even because of changes in technology. The problem becomes even more complicated if the objectives pertaining to demand and supply are multiple and conflicting in nature. Therefore, this paper deals with regulation of water resources system based on "if-then" fuzzy logic-based rules which interlinks concepts of interpolative reasoning, logical implications and certain inference tools to infer knowledge about a water resource system using linguistic descriptions. Reasonable inferences have been drawn using concept of tautologies viz. modulus ponens and modulus tollens. Finally, the model is applied to a practical case study in order to demonstrate effectiveness of the proposed logic. The main motive of this study is to demonstrate applicability of fuzzy inference system for regulating operations of water resource systems.
\end{abstract}

Keywords Approximate reasoning $\cdot$ Amaravathi dam $\cdot$ Inflow $\cdot$ Outflow $\cdot$ Storage $\cdot$ Reservoir planning $\cdot$ Fuzzy logic $\cdot$ Fuzzy rule-based system: Mamdani and Sugeno methods

\section{Introduction}

Regulation of water resources system is an important task while formulating various important decision-making policies pertaining to planning, design, operation and maintenance of these systems. Each water resource system has its own kind of data collection and workflow techniques to define, review, identify, verify and execute efficient and effective operational plans. Several authors used different techniques to predict outcomes and regulate water resource systems by applying concepts of fuzzy logic. The notion of fuzzy set is the formation of conceptual framework for representation of a set of data at convenient point in many real-life applications. In general, a water resources system has a large set of data which require careful analysis for taking appropriate decisions pertaining to water allocation, quality and quantity management. These data should be reliable enough so that they can not only be used in optimal and justifiable manner but also should be communicated and

Ajit Pratap Singh

aps@pilani.bits-pilani.ac.in

1 Department of Civil Engineering, Birla Institute of Technology and Science, Pilani 333031, India processed effectively to all concerned stakeholders including end-users in an user-friendly manner.

A water resource system requires to reduce projected gaps between water supply and demand to fulfil future needs of water requirement for various beneficial uses. This is a complicated task that requires sound and reliable information about physical, hydrological and demographic characteristics of a water resource system/river basin. It is not only necessary to address complex socio-economic systems with different interest groups pursuing multiple and conflicting objectives but also deal inherent hydrological variability with uncertainty in data that complicate implementation of regulation policies of a water resource system. Recognizing these facts, several researchers have applied concepts of fuzzy logic, genetic algorithms (GAs) and artificial neural network (ANN) techniques in water resource management to overcome these uncertainties. The concepts of fuzzy logic have been used by assuming that it is a super set of conventional (or Boolean) logic. The traditional Boolean logic uses 0 or 1 to describe the membership relation between one specific object and the class of this kind of objects. When the object belongs to that class, the value of the characteristic function that describes the membership relation between one specific object and classification is 1 , whereas the value is 
0 when the specific object doesn't belong to the class completely. Using fuzzy logic, it is easy to calculate the degree of an object to which it is a member. The grade of membership function can be achieved by adopting the concept of a membership function to assign a number ranging from zero (absolute not belonging) to unity (fully belonging) according to the degree of belongingness to each element.

In this paper, regulation policies of a water resources system has been formulated using concepts of fuzzy logic to incorporate uncertainty due to vagueness. The vagueness can be defined by deriving appropriate membership functions. The nature of uncertainty decides the fuzzy set to be used for representing the vagueness or uncertainty of the system. Multiple degrees of membership can prove to be key in decision-making process where infinite number of values between two extreme points exists. This paper deals with the application of fuzzy logic as a domain tool for the representation of the reservoir regulation problem by a set of predefined rules as specified by the decision maker. Finally, a case study has been presented as an extension of the operation to understand the characteristics and efficiency of the water resources system of Amaravathi dam located on the Amaravathi river under fuzzy environment to depict a general framework for regulating water resources systems and arriving at decisions and policies for water resources systems. Scenario analyses using concepts of fuzzy logic have identified/obtained various important/critical indicators to overcome uncertainties involved in water resource management. These indicators are useful to test the performance of alternative management policies which are very essential in dealing with complicated planning water resources problems and could help decision makers to obtain viable alternatives under uncertainty.

\section{Literature review}

Fuzzy set was introduced after Lotfi Zadeh (1965) and further developed by many authors. Fuzzy set is used to describe the lack of distinction between events occurring in a universe of discourse (i.e. the universe of all available information about a given problem). Zadeh has extended the idea of binary membership, i.e. no membership or full membership to "degree of membership" functions, where an element may consist of infinite number of values in between endpoints representing various degrees of membership. Degree of membership used in fuzzy logic concepts is function obeying plausible properties such as monotonicity, normality and symmetry. A fuzzy set can have a number of membership functions unlike a crisp set which has unique membership function. Thus, fuzzy sets are flexible in maximizing membership functions for a particular application and utility (Ross 2010). Several authors have used the concepts of fuzzy sets and fuzzy logic applications in combination with other soft computing techniques. Panigrahi and Mujumdar (2000) have applied fuzzy inference system (FIS) as a tool to optimize operations of reservoir systems. The application of fuzzy in spatial decision support system (SDSS) was enumerated by combining geographical information system (GIS) with particular objectives in water distribution networks (Makropoulos et al. 2003). Singh and Ghosh (2003a, b) have developed a fuzzy-based conceptual modelling framework for water quality management of a river basin. They also incorporated uncertainty aspects of river basin water quality management using Monte Carlo simulation analysis (2003b). Fuzzy logic in the application of material characterization and defect detection was introduced by Khanfar et al. (2003). Cimen and Saplioglu (2007) have evaluated flow of water through hydraulic structures using fuzzy logic concepts. Sert et al. (2007) have applied fuzzy multi-objective optimization for releasing water from reservoir for various beneficial uses. Altunkaynak et al. (2005) have demonstrated application of Takagi-Sugeno (TS) method to predict consumption rate of water in Istanbul, Turkey. Singh et al. (2007) have presented interactive fuzzy multi-objective linear programming (IFMOLP) model to maximize water quality of river Yamuna under optimum treatment cost.

A number of studies have also been presented to incorporate uncertainty in assessing water resources potential, water allocation, water quality, reservoir operation and allied field of study using multi-criteria decision-making methods (Chan et al. 2009; Flug et al. 2000; Mahmoud and Garcia 2000; Singh 2008; Singh and Shrivastava 2014; Singh et al. 2017; Srdjevic et al. 2004; Teodorovic 1999). Talon et al. (2014) enumerated the performance modelling on longrange collection of data using evidence theory and fuzzy logic. Srinivas et al. (2015) have presented fuzzy inference tool to develop a model for assessing groundwater quality in Bikaner district in Rajasthan, India. They considered various water quality parameters to evaluate water quality status in groundwater wells located in the region. They ranked suitability of these wells corresponding to drinking and irrigation uses so that decision makers can formulate appropriate policies for sustainable planning of groundwater resources with optimal treatment options in the region.

Though concepts of fuzzy logic have been applied in various water-related problems, above literature review clearly demonstrates that there has been a definite requirement for developing appropriate regulation policies for reservoir operations/management in formulating/acquiring sustainable development strategies for water resources systems especially under varying climatic and hydrological conditions in Indian context. 


\section{Background of the study}

The concepts of fuzzy logic have been used in real-life applications of engineering and social sciences. A number of studies in the literature enumerates the application and usage of fuzzy logic as a tool for associating objective and subjective nature of a problem. This paper develops a general framework for regulating water resources systems to formulate appropriate policies for scientific decisions using fuzzy inference rule-based systems. It evaluates outflow of the system, with respect to a given set of input variables such as inflow (I), storage (S) and time of period $(\mathrm{T})$, under varying climatic and hydrological conditions. Although the model is intended for the entire basin, due to lack of adequate and reliable data, the main targets of the study were limited to a practical case study on Amaravathi dam to demonstrate application of the proposed framework. This dam was constructed on Amaravathi river, which is one of the major tributaries of the river Cauvery, originating from western Ghats near Moonar at about 1554 metres above mean sea level (MSL). Amaravathi river system facilitates for double crops mainly paddy and sugarcane. The dam is located with latitude and longitude of $10^{\circ} 24^{\prime} 55.86^{\prime \prime}$ North and $70^{\circ} 15^{\prime} 49.3^{\prime \prime}$ East, respectively, which covers a vast catchment area of $829,440,000$ square metre. The periodic inflow for this river system is contributed by monsoon rainfall which is generally received from both south-west Monsoon and north-east Monsoon though north-east Monsoon contributes higher rainfall/ inflow than south-west monsoon. Amaravathi dam envisages stabilizing of existing irrigation system which also provides additional irrigation facilities to the surrounding districts of Tamil Nadu. The live storage capacity (LSC) of the dam is 110 million cubic metre (MCM). The inflow, storage and outflow of the dam change periodically due to change in demand which in turn depends upon method of irrigation, water usage habits by the stakeholders, type of crop cultivated, prevailing atmosphere and temperature, evaporation and requirement of hydropower generation. In order to control and increase the efficiency, different kinds of mathematical optimization techniques have been devised, which in turn controls, regulates and optimizes the outflow of any stream. However, the huge data requirement and inter-dependencies of various decision variables have made these techniques very complicated. The problem becomes further aggravated due to uncertainty and ambiguity associated with data measurement and its prediction that can be treated well using concepts of fuzzy logic. The subsequent sections of the paper explain about the problem formulation to regulate outflow in reservoir system in general and a practical case study of Amaravathi dam specifically wherein the inflow, storage and outflow variables are simulated under different conditions. The study has delineated the optimal outflow on the basis of three common methods for deductive inference of fuzzy rule-based system: Mamdani method, Sugeno models and Tsukamoto models. The results obtained from these methods were compared to derive conclusive recommendations for an accurate outflow.

\section{Problem formulation}

There are various techniques which have been used to describe uncertainties associated with a particular problem. They are possibility theory, evidence theory, imprecise probabilities theory, fuzzy sets etc. For a water resource system, water storage, inflow and outflow can be expressed as fuzzy sets due to their uncertain and ambiguous characteristics. The flow and storage data applicable for a particular dam or reservoir can be discretized for a given period. Panigrahi and Mujumdar (2000) have divided annual flow of reservoir systems into thirty-six periods in their study. Each period with the mean value of 10 days flow and storage, and last six periods were added with extra 1 day in order to compensate for 366 days in a year. Application of nonlinear mathematical approach used conventionally was difficult, when analysis and modelling were made for complicated systems that too with limited information on past data. In turn, fuzzybased analysis can be used as a tool for modelling of waterrelated problems for varied field applications as discussed in the literature review. Modelling of a fuzzy system basically involves identification of premises and consequences of the system, discretization of discourse of universe into subsequent sets, specifying linguistic terms for the identified antecedent and consequent for each sets. Each set could be associated with a membership function (MF). Different kinds of membership function could be specified based on our system. Selection of specific kind of membership functions for modelling depends upon the intensive experimental analysis which in turn requires extensive data. The degree of membership functions can be derived using various techniques available in the literature such as inductive reasoning, entropy minimization screening method, batch least square algorithm, gradient methods, learning from examples and previous studies. A general fuzzy system consists of fuzzifier, rules, inference engine and defuzzifier as shown in Fig. 1.

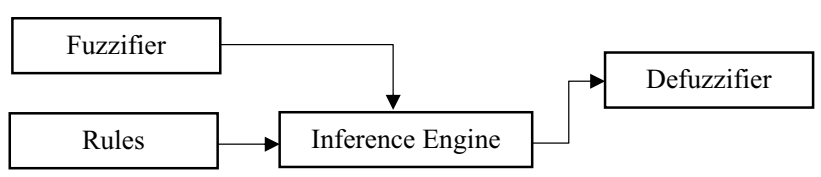

Fig. 1 Fuzzy logic system 
For a water resource system, various membership functions have been used to classify linguistic terms to apply proper rule base and discretization process. They are triangular, trapezoidal, bell-shaped, Gaussian etc., which generally obeys monotonicity, normality and symmetry characteristics. Each kind of MF's leads to different choice of overlap among different sets in a universe of discourse. For example, inflow could have different sets such as medium inflow (MI) and medium-high inflow (M-HI) which may overlap with different degree. The degree of overlap between different sets decides the membership value of inflow for a given set of antecedents. Based on the number of antecedents and consequents, fuzzy logic system could be named as $x$-input and $y$-output system. The proposed water resources system has been considered as three-input and single-output system. The membership function and classification for inflow, outflow and storage were derived using methods available in the literature. Some of the methods used for classification and developing fuzzy models were inductive reasoning, batch least squares algorithm (BLS), recursive least squares algorithm (RLS), gradient method (GM), learning from examples (LFE), modified learning from example (MLFE) and clustering method (CM). Cardinality or cardinal number is a metric which is useful in defining attribute of sets on the universe on which the sets were defined. Cardinal number gives the total number of elements in the universe. The collection of different elements in a universe combines to form a set. Power set is one which accommodates all possible collection of sets in the universe. The sets formed by collection of elements were also called as set theoretic forms. The number of universe of discourse and sets defined was based on the definition of the problem. In this study, water resources systems have four universe of discourse for inflow, storage and time of period considered as input and outflow as output. Each universe of discourse was divided into number of sets based on required number of membership functions (MF's). The universe of inflow, storage and outflow were characterized based on the average of historical inflow, outflow and storage details of the dam. The sets were represented in linguistic terms in order to form appropriate rule base and inference system. In a universe, to define the membership of an element belongs to a set, characteristic functions or indicator functions were used. The characteristic function signifies the degree to which an element belongs to different sets defined in a universe which also attempts to describe vagueness and ambiguity that a particular set's extent of boundary. Based on ample literatures, fuzzy classification and development methods range of membership functions (MF) for each universe of discourse were fixed. Figure 2 shows the range of membership function and its distribution for the universe of discourse inflow for the present case study of Amaravathi dam.

Figure 3 depicts range of membership function and its distribution for the universe of discourse storage, whereas Fig. 4 shows characteristics of membership function for the universe of discourse outflow for the present case study. The range and distribution of membership function for time of period have been considered as trapezoidal and triangular form in order to account for a particular period of flows and storage during a specified year. As mentioned earlier, each element from the universe of a fuzzy set was mapped to the universe of membership values using a function-theoretic

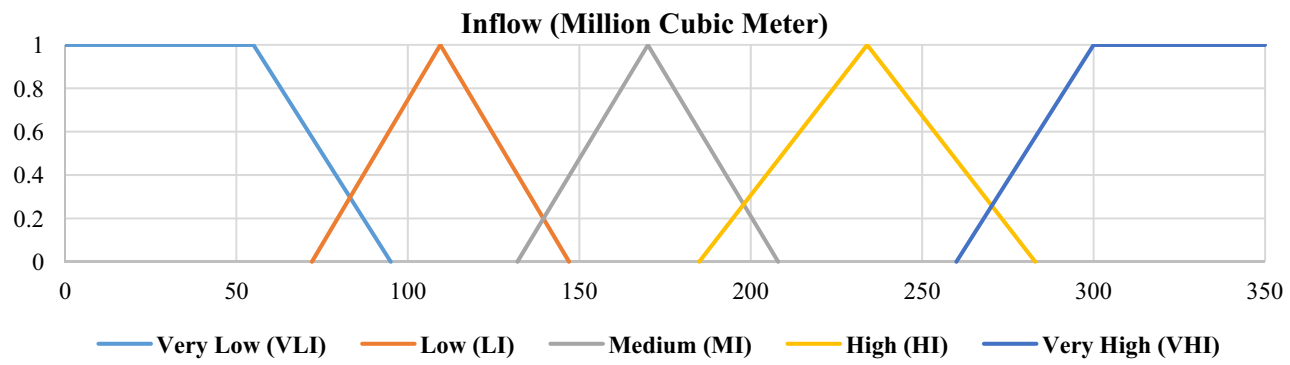

Fig. 2 Membership function for the universe inflow

Fig. 3 Membership function for the universe storage

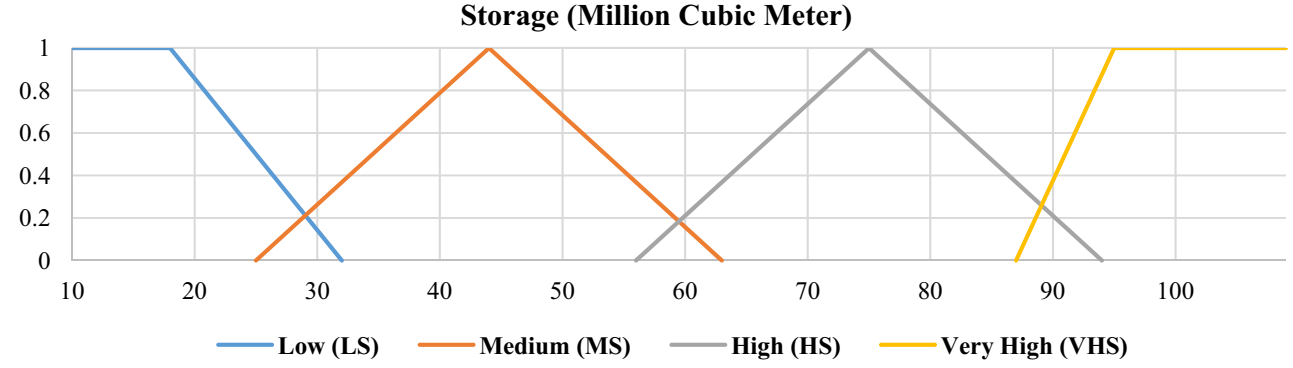




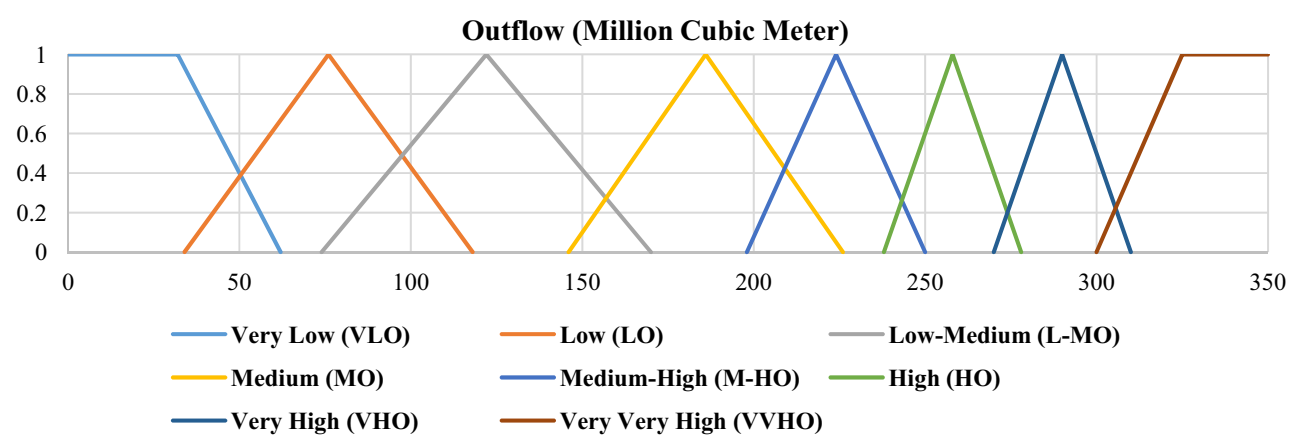

Fig. 4 Membership function for the universe outflow

Table 1 Membership function and extent-universe inflow (I)

$$
\begin{aligned}
& \mu_{\mathrm{VLI}}=\left\{\begin{array}{c}
1.00 \leq x \leq 55 \\
-0.025 x+2.37555 \leq x \leq 95
\end{array}\right\} \\
& \mu_{\mathrm{LI}}=\left\{\begin{array}{c}
0.0267 x-1.9272 \leq x \leq 109.5 \\
-0.0267 x+3.92109 .5 \leq x \leq 147
\end{array}\right\} \\
& \mu_{\mathrm{MI}}=\left\{\begin{array}{c}
0.0263 x-3.474132 \leq x \leq 170 \\
-0.0263 x+5.474170 \leq x \leq 208
\end{array}\right\} \\
& \mu_{\mathrm{HI}}=\left\{\begin{array}{c}
0.02041 x-3.78185 \leq x \leq 234 \\
-0.02041 x+5.78234 \leq x \leq 283
\end{array}\right\} \\
& \mu_{\mathrm{VHI}}=\left\{\begin{array}{c}
0.025 x-6.5260 \leq x \leq 300 \\
1300 \leq x \leq 350
\end{array}\right\}
\end{aligned}
$$

Table 2 Membership function and extent-universe storage (S)

$$
\begin{aligned}
& \mu_{\mathrm{LS}}=\left\{\begin{array}{c}
1.010 \leq x \leq 18 \\
-0.07143 x+2.285718 \leq x \leq 32
\end{array}\right\} \\
& \mu_{\mathrm{MS}}=\left\{\begin{array}{c}
0.05263 x-1.315825 \leq x \leq 44 \\
-0.05263 x+3.315844 \leq x \leq 63
\end{array}\right\} \\
& \mu_{\mathrm{HS}}=\left\{\begin{array}{c}
0.05263 x-2.947456 \leq x \leq 75 \\
-0.05263 x+4.947475 \leq x \leq 94
\end{array}\right\} \\
& \mu_{\mathrm{VHS}}=\left\{\begin{array}{c}
0.125 x-10.87587 \leq x \leq 95 \\
195 \leq x \leq 109
\end{array}\right\}
\end{aligned}
$$

form. This function maps fuzzy sets to a real numbered value on the interval 0-1. The inflow fuzzy sets were defined as VLI, LI, MI, HI and VHI on the universe inflow (I). Similarly LS, MS, HS and VHS were classified on the universe storage and VLO, LO, L-MO, MO, M-HO, HO, VHO and VVHO were classified on the universe outflow $(\mathrm{O})$. The corresponding characteristic function and extent of the above fuzzy sets on the universes inflow (I), storage (S) and outflow (O) are listed in Tables 1, 2 and 3, respectively, for the present case study. The relationship between different sets of universes was established to represent logical connectivity between various sets in the universes. As the present study
Table 3 Membership function and extent-universe outflow $(\mathrm{O})$

$$
\begin{aligned}
& \mu_{\mathrm{VLIO}}=\left\{\begin{array}{c}
1.00 \leq x \leq 32 \\
-0.033 x+2.06732 \leq x \leq 62
\end{array}\right\} \\
& \mu_{\mathrm{LO}}=\left\{\begin{array}{c}
0.02381 x-0.809534 \leq x \leq 76 \\
-0.02381 x+2.809576 \leq x \leq 118
\end{array}\right\} \\
& \mu_{\mathrm{L}-\mathrm{MO}}=\left\{\begin{array}{c}
0.02083 x-1.5416774 \leq x \leq 122 \\
-0.02083 x+3.54167122 \leq x \leq 170
\end{array}\right\} \\
& \mu_{\mathrm{MO}}=\left\{\begin{array}{c}
0.025 x-3.65146 \leq x \leq 186 \\
-0.025 x+5.65186 \leq x \leq 226
\end{array}\right\} \\
& \mu_{\mathrm{M}-\mathrm{HO}}=\left\{\begin{array}{c}
0.03846 x-7.61538198 \leq x \leq 224 \\
-0.03846 x+9.6153822 \leq x \leq 250
\end{array}\right\} \\
& \mu_{\mathrm{HO}}=\left\{\begin{array}{c}
0.05 x-11.9238 \leq x \leq 258 \\
-0.05 x+13.9258 \leq x \leq 278
\end{array}\right\} \\
& \mu_{\mathrm{VHO}}=\left\{\begin{array}{c}
0.05 x-13.5270 \leq x \leq 290 \\
-0.05 x+15.5290 \leq x \leq 310
\end{array}\right\} \\
& \mu_{\mathrm{VVHO}}=\left\{\begin{array}{c}
0.04 x-12300 \leq x \leq 325 \\
1.0325 \leq x \leq 350
\end{array}\right\}
\end{aligned}
$$

deals with more than one universe of discourse, functiontheoretic aggregation and composition operations were carried out in relations for defining fuzzy sets of each universe in order to arrive at mapping between antecedents and consequents of the model. The relationship developed herein represents mapping of different sets of universes through composition operators which were used to form relations between different universe of discourse. Basic types of composition operators used for this study were max-min composition operator and max-product composition operator. In this problem, both kinds of operators were used and results were arrived which show similar same pattern of variation in outflow from the water resources system. The max-min operator introduced by Zadeh has been used to express the approximate and inductive reasoning of fuzzy decisionmaking of water resources system.

The fuzzy information on inflow, storage and outflow of reservoir system has been represented using well-structured 
fuzzy rules. The proportions of fuzzy rules express the logical connectivity. For example, if proportion $\mathrm{M}$ is assigned to a fuzzy set called "medium inflow (MI)", then truth value of the proportion $\mathrm{M}$ is represented by Eq. (1):

$T(M)=\mu_{\mathrm{MI}}(x)$, where $0 \leq \mu_{\mathrm{MI}} \leq 1$

Equation (1) indicates the degree of truth for the proportion $M: x \in \mathrm{MI}$ is equal to membership grade of $x$ in the fuzzy set MI. The process of arriving at inference and learning of the structure for a basic "if-then" rule-based system involves the application of "implication connective" between different proportions defined for the process. The implication connector works based on a basic principle of a tool "modus ponens". For example, IF inflow (I) is very high (VHI) and storage (S) is low (LS) and time period (T) is 1, THEN outflow $(\mathrm{O})$ is low (LO) is a rule, this relation can be translated into relation between sets as $R=(((\mathrm{VHI} \times \mathrm{LO}) \cup(-\mathrm{VHI} \times \mathrm{O})) \cap$ $((\mathrm{LS} \times \mathrm{LO}) \cup(-\mathrm{LS} \times \mathrm{O})) \cap((\mathrm{T} X \mathrm{LO}) \cup(-\mathrm{T} \times \mathrm{O})))$. For a different proportion of antecedents, consequent can be derived using the above relation $R$. If elements in each set of the universe are expressed as $u, v, w$ and $z$ corresponding to fuzzy sets very high (VHI) in the universe of inflow (I), low (LS) in the universe of storage (S), time period of 1 and low (LO) in the universe of outflow (O), respectively, then membership function for the relation $\mathrm{R}$ can be expressed as given in Eq. (2):

$$
\begin{aligned}
\mu_{R}(u, v, w, z)= & \max \left[\left(\mu_{\mathrm{VHI}}(u) \wedge \mu_{\mathrm{LS}}(v) \wedge \mu_{1}(w)\right),\right. \\
& \left.\left(\left(1-\mu_{\mathrm{VHI}}(u)\right) \wedge\left(1-\mu_{\mathrm{LS}}(v)\right) \wedge 1-\mu_{1}(w)\right)\right\}
\end{aligned}
$$

\section{Fuzzy rule-based systems}

The outcome of fuzzy analysis can be expressed using inferential method to represent human knowledge of the subject. Typically, conclusion (consequent) can be derived if the fact (hypothesis) is known. As mentioned previously, approximate reasoning for arriving at theoretical foundation of imprecise proportions is the ultimate goal of fuzzy logic. For reasoning approximately fuzzy rule-based system in the form of "IF-THEN", convenient antecedent-consequent form is used. All important inferences can be made from a set of canonical rule-based system. A typical representation of canonical form of a fuzzy rule-based system has been given in Table 4. If there are more than one antecedent, it requires conjunctive or disjunctive operator for linking them. The conjunctive operator and disjunctive operator follow operations of standard intersection and union operations, respectively. The regulation and optimization of water outflow from a water resources system require minimization of available resources based on given input variables such as inflow, storage and particular time period of the year. Generally, an intersection operator is used for minimization of flows and storage for a particular period of time.

Table 4 represents canonical form of fuzzy rule base applicable to water resources system at Amaravathi, where $A^{1}, A^{2},, A^{r}, B^{1}, B^{2}, \ldots, B^{r}, C^{1}, C^{2}, \ldots, C^{r}$, and $D^{1}, D^{2}, \ldots, D^{r}$. represent the set of antecedents in the universe of inflow (I), storage $(\mathrm{S})$, time period $(\mathrm{T})$ and outflow $(\mathrm{O})$, respectively. The multiple antecedents can be aggregated using conjunctive operator. For example, IF inflow is $A^{1}$ AND storage is $B^{1}$ AND time Period is $C^{1}$ THEN outflow is $D^{1}$ is a fuzzy rule in rule-based system. The aggregation of antecedents using conjunctive operator is given in Eq. (3). Assuming $R^{s}$ as new fuzzy subset

$R^{s}=A^{1} \cap B^{1} \cap C^{1}$

which can also be expressed in the form of membership function as given in Eq. (4):

$\mu_{R^{s}}(u, v, w)=\min \left[\left(\mu_{A^{1}}(u), \mu_{B^{1}}(v), \mu_{C^{1}}(w)\right)\right]$

In real-life situations, a rule-based system consists of more than one rule, therefore each rule in the rule-based system contributes in a different way to arrive at overall conclusion from the individual consequent. Thus, aggregation process is carried out to arrive at overall consequent using a disjunctive operator, which acts same as that of union operator as defined for a fuzzy set. A disjunctive operation used for aggregation of consequents is given in Eq. (5). Assuming $Y^{s}$ as aggregated fuzzy output,

$Y^{s}=D^{1} \cup D^{2} \cup D^{3} \cup \ldots \ldots \ldots D^{r}$

which can also be expressed in the form of membership function as given in Eq. (6):

$\mu_{Y^{s}}(z)=\max \left[\left(\mu_{D^{1}}(z), \mu_{D^{2}}(z), \mu_{D^{3}}(z), \ldots \ldots \ldots \ldots \mu_{D^{r}}(z)\right)\right]$

\section{Graphical techniques of inference}

The mathematical procedures can be more decisive, if a decision process can be formulated with less number of rules. If number of rules in the process of decisionmaking increases, computation quickly becomes quiet onerous. The graphical form of inference has been quite
Table 4 The canonical form for a fuzzy rule-based system

\author{
Rule1 : IF Inflow is $A^{1}$ and Storage is $B^{1}$ and Time of Period is $C^{1}$ THEN Outflow is $D^{1}$ \\ Rule 2 : IF Inflow is $A^{2}$ and Storage is $B^{2}$ and Time of Period is $C^{2}$ THEN Outflow is $D^{2}$ \\ ... \\ Rule $r$ : IF Inflow is $A^{r}$ and Storage is $B^{r}$ and Time of Period is $C^{r}$ THEN Outflow is $D^{r}$
}


useful in order to increase the computation efficiency. Jang et al. (1997) have suggested three common methods for deductive inference of fuzzy rule-based system: Mamdani method, Sugeno models and Tsukamoto models. For example, if a fuzzy rule system is expressed as

Rule1 : IF Inflow is High (HI) and

Storage is High (HS) and Time Period is 1

THEN Outflow is Low - Medium(L - MO)

Rule2 : IF Inflow is Medium (MI) and Storage

is Medium (MS) and Time Period is 2

THEN Outflow is Medium(MO)

where High (HI), High (HS), 1, Medium (MI), Low (LS) and 2 represent fuzzy sets of rule base 1 and 2 of the system, respectively. Two different cases of Mamdani method and Sugeno models have been used for inferring the outcome in the graphical technique. Figure 5 shows the graphical Mamdani (max-min) inference representation, where the symbols A11 and A12 refer as the antecedents part of the first fuzzy rule and symbol B1 indicates the consequent part of fuzzy first rule. Similarly, A21 and A22 refer to the antecedents of the fuzzy second rule and symbol B2 indicates the consequent of the fuzzy second rule. All antecedents were connected by conjunctive rule "AND" so minimum membership value from the antecedent truncates the membership function of the consequent.
Also, the minimum membership value from the antecedent truncates the membership function of consequent for the second rule. Based on the connector used for aggregation of consequent membership function, the complete aggregation process has been carried out. The disjunctive (max) aggregation results have been obtained in the overall outer envelope of the aggregated membership function after combing rules 1 and 2 . The defuzzification process has been carried out in order to get the final crisp output value of the aggregated membership function using weighted average method. The max-product implication technique has also been used on similar lines in which during truncation process instead of truncating the consequent membership function, scaling of the corresponding membership function takes place. It finally results the reduced size of the membership function as shown in Fig. 6. Both process of Mamdani (max-min) and (max-product) inference and implication process has produced almost comparable output after defuzzification process.

The Sugeno method was also applied to arrive at the regulation of outflow of water resource system. In fact, Sugeno method applied for the practical case study has produced more accurate regulatory flow as compared to Mamdani method. The basic definition, which differs in Sugeno method, is that output is represented as a function of inputs with following form:
Fig. 5 Graphical Mamdani (max-min) inference method
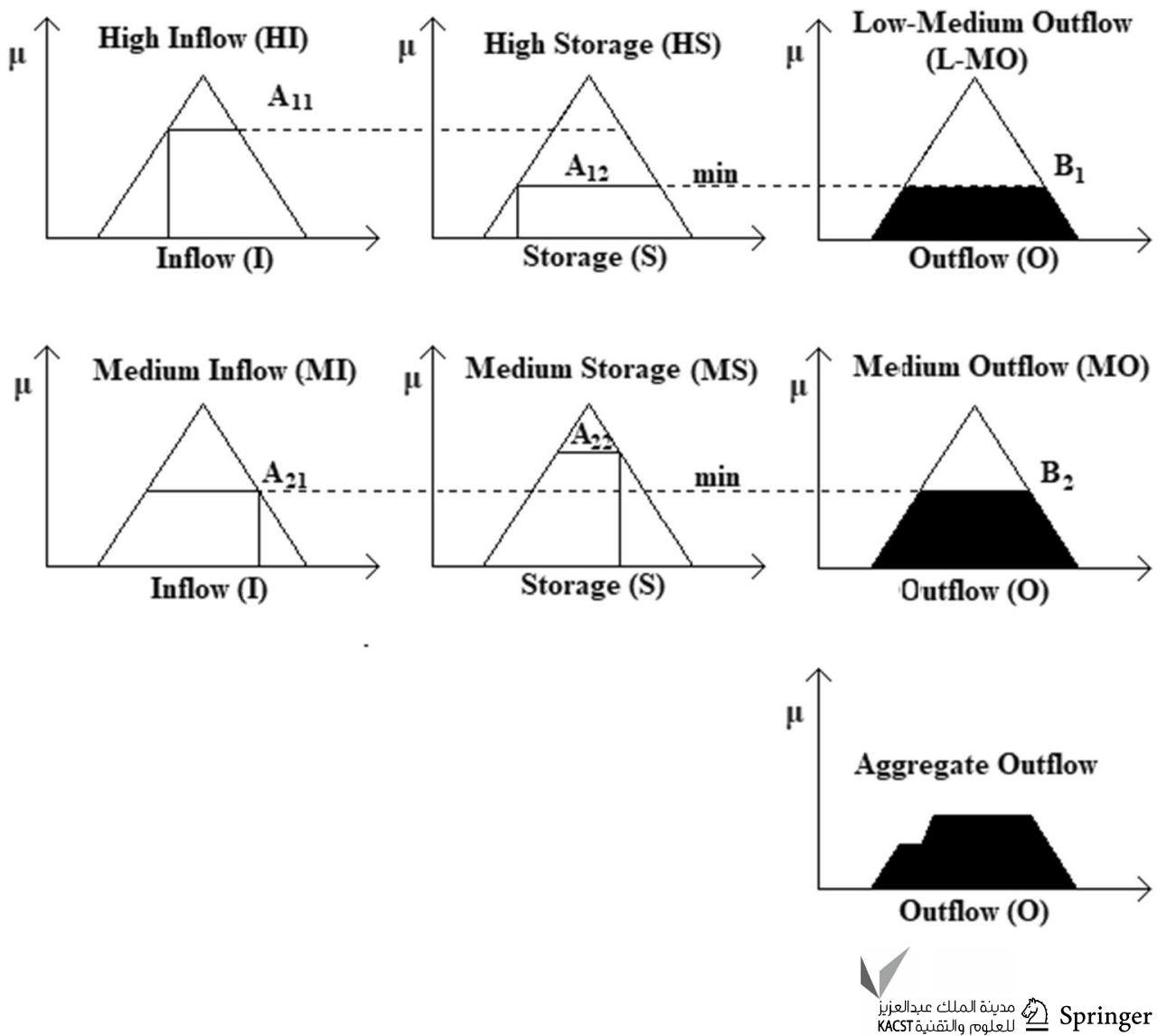
Fig. 6 Graphical Mamdani (max-product) implication method
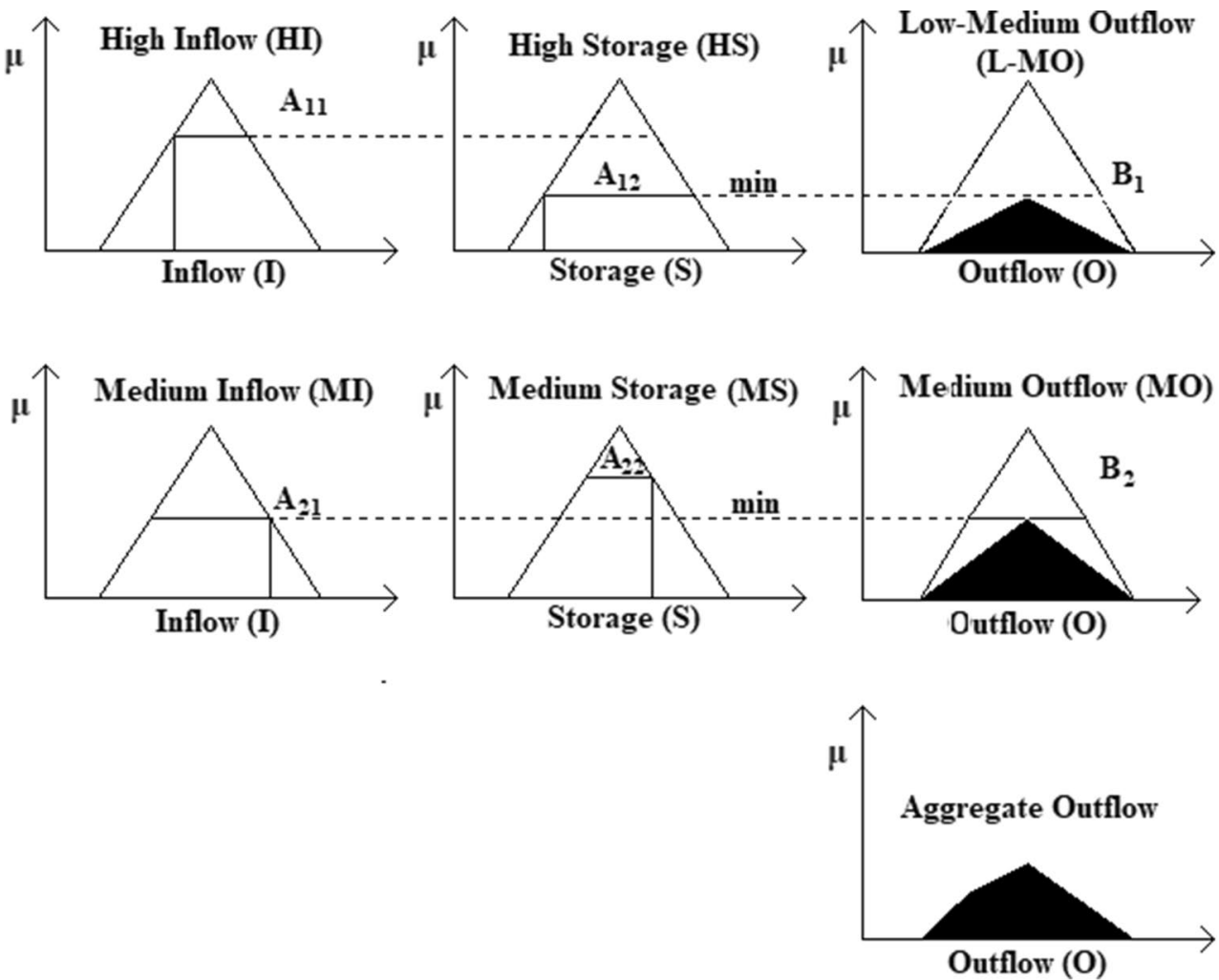

IF Inflow is High(HI) and Storage

is High(HS) and Time of Period is 1.

THEN Outflow is Low - Medium(L - MO) $=\mathrm{f}(\mathrm{HI}, \mathrm{HS}, 1)$.

The Sugeno method with constant function is a special case of Mamdani method which is also called as zero-order Sugeno model. The behaviour of any fuzzy system can be determined by the degree of overlap between the membership function of the antecedents that determines the smoothness of the system. In the Sugeno method, each rule has a crisp output, so arrival at the aggregated output by defuzzification process is much easier than the Mamdani method. In this study, weighted average method of defuzzification process has been used to get the crisp values.

\section{Case study: Amaravathi dam}

Above-described general framework for the regulation of water resource system was applied to Amaravathi dam to study the effectiveness and efficiency of the framework. Details of inflow, outflow and storage were collected from Tamil Nadu Water Supply and Drainage (TWAD) Board. 4 years of flow data and storage data were collected. A fuzzy rule-based system was derived based on the classification of available data. The system of fuzzy relational equations was derived based on the aforementioned membership classification, set classification, relational operations and connectors. $R^{r}$ has been taken as fuzzy relations where $\mathrm{r}$ denotes the number of total relations that defines the interconnection between fuzzy sets in the universe of discourse inflow (I), storage (S), time period (T) and outflow (O). A total of 36 periods were considered annually with each period averages 10 days flows and storages and last six periods averages 11 days in order to compensate for 366 days of a specified year. Let $u, v, w$ and $z$ be the elements in the universe inflow (I), storage (S), time period (T) and outflow $(\mathrm{O})$, respectively, then fuzzy relational equation has been expressed as

$$
\begin{aligned}
& \tilde{R}^{1}: z^{1}=\left(u_{1^{1}} \circ v_{1^{2}} \circ w_{1^{3}}\right) \circ \tilde{R}^{1} \\
& \tilde{R}^{2}: z^{2}=\left(u_{2^{1}} \circ v_{2^{2}} \circ w_{2^{3}}\right) \circ \tilde{R}^{2} \\
& \tilde{R}^{3}: z^{3}=\left(u_{3^{1}} \circ v_{3^{2}} \circ w_{3^{3}}\right) \circ \tilde{R}^{3} \\
& \ldots \\
& \tilde{R}^{r}: z^{r}=\left(u_{r^{1}} \circ v_{r^{2}} \circ w_{r^{3}}\right) \circ \tilde{R}^{r}
\end{aligned}
$$

Total number of 108 rules were derived from the existing data where $r=108$ and $u_{r^{1}}, v_{r^{2}}, w_{r^{3}}$. were data sets. The proposed fuzzy framework has been to derive following aggregated equation:

$$
\begin{gathered}
z=\left(\left(u_{1^{1}} \circ v_{1^{2}} \circ w_{1^{3}}\right) \circ \tilde{R}^{1}\right) \mathrm{OR}\left(\left(u_{2^{1}} \circ v_{2^{2}} \circ w_{2^{3}}\right) \circ \tilde{R}^{2}\right) \mathrm{OR} \ldots \\
\operatorname{OR}\left(\left(u_{r^{1}} \circ v_{r^{2}} \circ w_{r^{3}}\right) \circ \tilde{R}^{r}\right) \mathrm{z}=(\text { uovow }) \circ \tilde{R}
\end{gathered}
$$


Table 5 Membership functions classification and range

\begin{tabular}{|c|c|c|c|c|c|c|c|c|}
\hline \multicolumn{3}{|c|}{ Inflow (I) (MCM) } & \multicolumn{3}{|c|}{ Storage (S) (MCM) } & \multicolumn{3}{|c|}{ Outflow (O) (MCM) } \\
\hline & \multicolumn{2}{|c|}{$q$ varies 1 to 5} & & \multicolumn{2}{|c|}{$m$ varies 1 to 4} & & \multicolumn{2}{|c|}{$p$ varies 1 to 8} \\
\hline & Notation & Range & & Notation & Range & & Notation & Range \\
\hline 1 & VLI & 0-95 & 1 & $\mathrm{LS}$ & $10-32$ & 1 & VLO & $0-62$ \\
\hline 2 & LI & $72-147$ & 2 & MS & $25-63$ & 2 & LO & $34-118$ \\
\hline 3 & MI & $132-208$ & 3 & HS & $56-94$ & 3 & L-MO & $74-170$ \\
\hline 4 & $\mathrm{HI}$ & $185-283$ & 4 & VHS & $87-109$ & 4 & MO & $146-226$ \\
\hline \multirow[t]{4}{*}{5} & VHI & $260-350$ & & & & 5 & M-HO & $198-250$ \\
\hline & & & & & & 6 & $\mathrm{HO}$ & $238-278$ \\
\hline & & & & & & 7 & VHO & $270-310$ \\
\hline & & & & & & 8 & VVHO & $300-350$ \\
\hline
\end{tabular}

where $\tilde{R}$ is defined by $\tilde{R}=\tilde{R}^{1} \cup \tilde{R}^{2} \cup \ldots \tilde{R}^{r}$.

The canonical rule base for the above case study has been expressed as

$R^{r}=\mathrm{IF}$ Inflow is $A^{q}$ AND Storage is $A^{m}$ AND Time of Period is $A^{n}$

\section{THEN Outflow is $\mathrm{B}^{\mathrm{p}}$}

where $r=108$ and $q, m$ and $p$ vary as given in Table 5 and $n$ varies from 1 to 36 . The membership function ranges and classification have been obtained using inductive reasoning, batch least square algorithm (BLS), recursive least square algorithm (RLS), learning from examples (LFE) and modified learning from examples (MLFE).

\section{Results and discussion}

MATLAB was used for modelling of water resources system presented in this paper. The systems process has been identified by set of 108 rules. A new set of inputs alone, i.e. inflow, storage and time period, was given as input for time period of 36 and outflow was evaluated as the output. The results were compared with the original outflow of the system. Both forms of Mamdani inference methods viz., max-min method and max-product method were used to obtain outflow of the system. It has been found that the outflow of the system obtained by Mamdani inference method is in good relationship with the actual outflow as shown in Fig. 7. It was observed that the outflow obtained from max-min inference method is same as that determined from max-product inference method.

It is also interesting to note that outflow obtained from Sugeno constant method predicts outflow regulation very well for the given set of inputs. An average relative error percentage (AREP) was calculated for both Mamdani method and Sugeno constant model in order to consider the overall periodic prediction and regulatory deviation. The AREP values were obtained as 0.89 and 0.14 for Mamdani method and zero-order Sugeno model, respectively.

This paper presented a generic fuzzy inference system (FIS) that can be used to model operation of a reservoir and that can be used as an implementable reservoir policy. Previous studies that used FIS as a technique to model operation of a reservoir utilized models such as stochastic dynamic programming (SDP) that can be used as a base of knowledge for formulating fuzzy rules. In the present study, fuzzy rules were formulated based on the past data
Fig. 7 Outflow comparisonactual and fuzzy inference

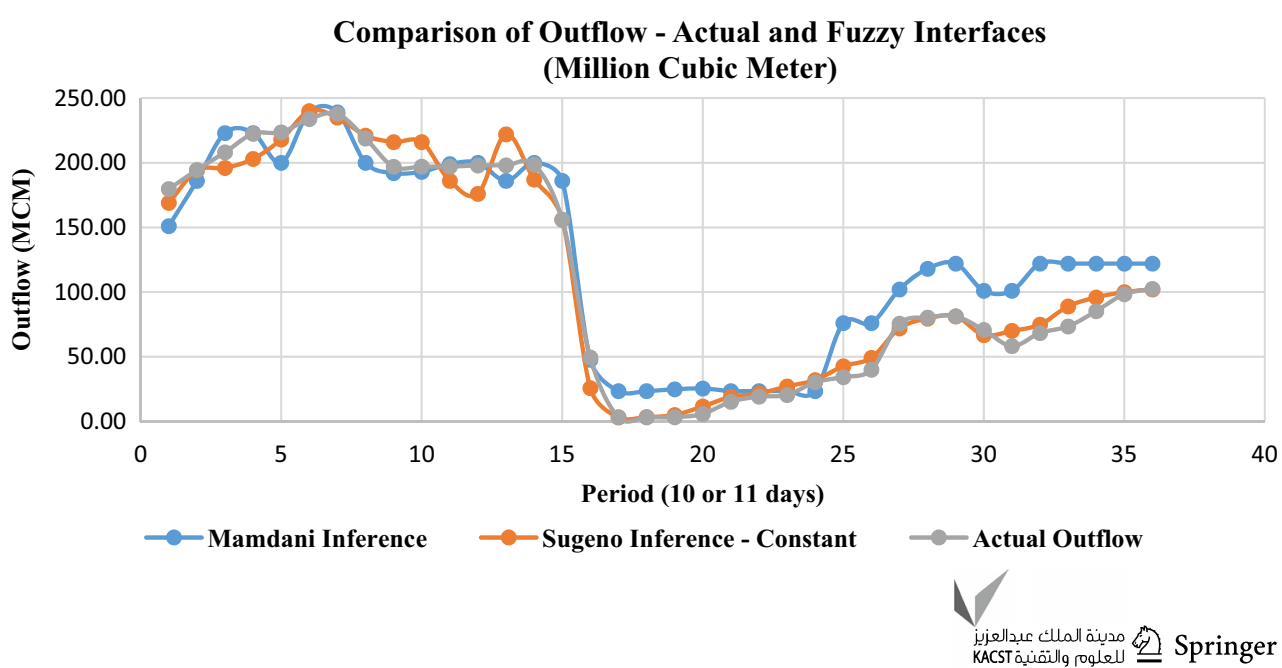


available from the particular dam itself. In general, fuzzy rules that are arrived based on utilization of conventional optimization techniques such as SDP cannot be substituted, even though formulating FIS based on a particular dam can offer a chance for reservoir operators to involve in formulating the rule base for FIS. In this way, utilization of FIS may increase the participation of local authorities and personnel to arrive at the policies that are more acceptable when compared to the policies that are arrived based on more complicated optimization models. However, FIS has limitations of dimensionality problems due to increase in number of fuzzy sets.

\section{Conclusions}

A generic FIS was formulated in this study in order to understand its applicability to regulate water resources system. FIS formulated in this study utilizes past data from the dam itself in order to arrive at the knowledge base to formulate fuzzy rules. Utilization of past data and transferring the data to fuzzy rules could cause effective participation of local authorities itself. Increase in the participation of local authorities and personnel could result in enhanced and best utilization of water resources. Different needs can be satisfied by implementing simplistic approaches such as FIS described in this study.

Results obtained from Mamdani inference method and Sugeno inference constant model suggest satisfactory regulation of water for the case study of Amaravathi dam. Further, Sugeno first-order model was obtained by relating outflow as a function of inflow, storage and time period. A linear equation obtained using genetic algorithm (GA) as a tool provides a large difference with the AREP value as of 2.72. The outflow obtained using Sugeno constant model has been found with very good correlation as compared to the Mamdani method with actual regulatory policies carried for outflows in the dam. The Sugeno model also reduces time required for aggregation of consequents. In the Sugeno model, each rule has a crisp output, so aggregation of output was done by weighted average method to obtain overall output of the system. Further regulation and optimization can be obtained by accumulation of further knowledge of the system to attain the maximum wisdom of the system. The framework developed in this paper explains about the problem formulation to regulate outflow in a reservoir system in general and a case study of Amaravathi dam specifically wherein the inflow, storage and outflow variables are simulated under different conditions. The study has delineated the optimal outflow on the basis of three common methods for deductive inference of fuzzy rule-based system: Mamdani method, Sugeno models and Tsukamoto models. The results obtained from these methods were compared to derive conclusive recommendations for an accurate outflow which will be very useful to assess performance of different alternatives to be adopted for allocation and transfer of water from the dam for different beneficial usages under economic, social and environmental constraints.

Even though, this study has developed a generic model to regulate water resources system. The results obtained is sensitive to the type of membership function utilized. Thus, FIS has limitations of dimensionality problems due to increase in number of fuzzy sets. This opens up future scope of studies to define appropriate membership functions and defuzzification methods.

Open Access This article is distributed under the terms of the Creative Commons Attribution 4.0 International License (http://creativeco mmons.org/licenses/by/4.0/), which permits unrestricted use, distribution, and reproduction in any medium, provided you give appropriate credit to the original author(s) and the source, provide a link to the Creative Commons license, and indicate if changes were made.

\section{References}

Altunkaynak A, Ozger M, Cakmekci M (2005) Water consumption prediction of Istanbul city by using fuzzy logic approach. Water Resour Manage 19:641-654

Chan APC, Chan DWN, Asce M, Yeung JFY (2009) Overview of the application of fuzzy technique in construction management research. J Constr Eng Manag 135:1241-1252

Cimen M, Saplioglu K (2007) Stream flow forecasting by fuzzy logic method. In: International congress on river basin management, pp 612-620

Flug M, Seitz LH, Scott JF (2000) Multicriteria decision analysis applied to Glen Canyon Dam. J Water Resour Plann Manag 126:270-276

Jang J, Sun C, Mizutani E (1997) Neuro-Fuzzy and soft computing: a computational approach to learning and machine intelligence, 2nd edn. Pearson Education, Inc., New Jersey

Khanfar A, Abu-khousa M, Qaddoumi N (2003) Microwave near-field nondestructive detection and characterization of dis bonds in concrete structures using fuzzy logic techniques. Compos Struct 62:335-339

Mahmoud MR, Garcia LA (2000) Comparison of different multicriteria evaluation methods for Red Bluff diversion dam. Environ Modell Softw 15:471-478

Makropoulos CK, Butler D, Maksimovic C, ASCE A (2003) Fuzzy logic spatial decision support system for urban water management. J Water Resour Plann Manag 129:69-77

Panigrahi DP, Mujumdar PP (2000) Reservoir operation modelling with fuzzy logic. Water Resour Manage 14:89-109

Ross TJ (2010) Fuzzy logic with engineering applications, 3rd edn. Wiley, New York

Sert M, Opan M, Temiz T (2007) Multiobjective optimal planning in multiple reservoir systems. In: International congress on river basin management, pp 554-567

Singh AP (2008) An integrated fuzzy approach to assess water resources' potential in a watershed. J Comput Math 1:7-23

Singh AP, Ghosh SK (2003a) Conceptual modelling and management of water quality in a river basin. In: Ramanathan AL, Ramesh (ed) Recent trends in hydrogeochemistry, Capital Books, New Delhi, pp 207-220

Singh AP, Ghosh SK (2003b) Uncertainty analysis in river basin water quality management. In: Raju KS, Sarkar AK, Dash M (eds) 
Integrated water resources planning and management. Jain Brothers, New Delhi, pp 235-246

Singh AP, Shrivastava P (2014) A comparative study on water quality assessment of a river using AHP and PROMETHEE techniques. In: Proceedings of 19th international conference on hydraulics, water resources, coastal and environmental engineering (hydro 2014 international), December 18-20, 2014, Bhopal, pp 880-890

Singh AP, Ghosh SK, Sharma P (2007) Water quality management of a stretch of river Yamuna: an interactive fuzzy multi-objective approach. Water Resour Manage 21:515-532

Singh AP, Chakrabarti S, Kumar S, Singh A (2017) Assessment of air quality in Haora river basin using fuzzy multiple-attribute decision making techniques. Environ Monit Assess 189:373. https://doi. org/10.1007/s10661-017-6075-3

Srdjevic B, Medeiros YDP, Faria AS (2004) An objective multi-criteria evaluation of water management scenarios. Water Resour Manage $18: 35-54$
Srinivas R, Bhakar P, Singh AP (2015) Groundwater quality assessment in some selected area of Rajasthan, India using fuzzy multi-criteria decision making tool. ICWRCOE'15. Aquatic Proced 4:1023-1030

Talon A, Curt C, Boissier D (2014) Performance assessment based on evidence theory and fuzzy logic: application to building and dam performance. J Comput Civil Eng 28:124-133

Teodorovic D (1999) Fuzzy logic systems for transportation engineering: the state of the art. Transp Res Part A 33:337-364

Zadeh LA (1965) Fuzzy sets. Inf Control 8:338-353

Publisher's Note Springer Nature remains neutral with regard to jurisdictional claims in published maps and institutional affiliations. 